\title{
Parental knowledge, attitudes and perceptions regarding infant basic life support
}

Patricia Ching Yen $\underline{\text { Chia }}^{1}$, MBChB, GDFM, Wee Bin $\underline{\operatorname{Lian}}^{2,3,4}$, MMed, MRCP

INTRODUCTION Out-of-hospital cardiopulmonary arrest (CPA) in children is rare but significant, with poor survival rates and high morbidity. Asystole is the most common dysrhythmia, and cardiopulmonary resuscitation (CPR) is of great importance in such cases. We aimed to survey the knowledge, attitudes and perceptions of parents in Singapore regarding infant basic life support (IBLS).

METHODS A questionnaire survey was administered to parents of children managed at the Neonatal Department of Singapore General Hospital, Singapore, between 1 September and 31 December 2008. The questionnaire consisted of three sections - section A collected demographic data, section B included questions on knowledge, and section $\mathrm{C}$ explored attitudes and perceptions. Knowledge T-scores were analysed for the entire cohort and subanalysed with respect to prior IBLS training.

RESULTS In our study cohort $(n=375)$, the median Basic Knowledge (BK) T-score was 7 (range 1-9) and the pass rate was 55\%. Median BK T-scores were significantly different between untrained (6; range $3-9$ ) and previously trained (8; range 3-9) participants. A majority of the trained participants obtained pass marks. Median Total Knowledge T-score, involving advanced questions, for previously trained participants was 11 (range 3-14), but pass rate was low (35.7\%). Higher educational qualification was a significant factor impacting all scores. Untrained participants indicated interest in attending IBLS courses, while trained participants were interested in refresher courses.

CONCLUSION IBLS training, as part of basic cardiac life support training, is important given that CPR can significantly alter the outcome in children with CPA. Our survey revealed knowledge gaps that could be bridged through formal training. Refresher courses to regularly update parents' knowledge are recommended.

Keywords: cardiopulmonary resuscitation, infant basic life support

\section{INTRODUCTION}

Out-of-hospital cardiopulmonary arrest (CPA) in children is relatively rare, with a reported annual incidence of 9.1-9.8 per 100,000 children aged less than 16-17 years. ${ }^{(1,2)}$ While its incidence has remained fairly constant over the years, ${ }^{(1,2)}$ a higher than usual rate of 19.2 per 100,000 children was reported in 1999 by Sirbaugh et al. ${ }^{(3)}$ In terms of personyears, studies from the United States, Canada and Netherlands have reported incidences ranging from 8.04 to 11.2 patients per 100,000 person-years. ${ }^{(4-6)}$ Despite its relatively low incidence, CPA in children is considered significant. According to Atkins et al, the incidence of CPA among the paediatric age groups was highest among infants (72.7 per 100,000 personyears for infants; 3.73 per 100,000 person-years for children; 6.37 per 100,000 person-years for adolescents). ${ }^{(5)}$

The outcome of CPA in children is reported to be generally very poor, with high rates of mortality and morbidity, and survival rates between $0 \%$ and $27 \% .{ }^{(1)}$ An earlier study from Singapore reported that only 4 out of 85 children who presented with out-of-hospital CPA to a children's emergency department survived to discharge, and all survivors had neurological deficits. ${ }^{(7)}$ Among the paediatric age groups, survival was the lowest among infants (3.3\%) when compared to children
$(9.1 \%)$ and adolescents $(8.9 \%) .{ }^{(5)}$ The most common causes of CPA were reported to be trauma, sudden infant death and respiratory disease, with asystole being the most common presenting dysrhythmia. . $^{(1,2,8)}$

A majority of paediatric CPA are reportedly unwitnessed, ${ }^{(1,7-9)}$ with bystander rates for cardiopulmonary resuscitation (CPR) usually reported to be low (range $17 \%-31 \%$ ). ${ }^{(1,2,7)}$ However, bystander CPR is consistently reported as a factor that significantly contributes to improved survival or hospital discharge rate in children with CPA.(2,9) Therefore, caregivers and potential bystanders should be educated on CPA and made aware of the benefits of CPR.

The objective of this survey was to determine the knowledge, attitudes and perceptions of parents in Singapore with regard to infant basic life support (IBLS), which is an integral part of training on basic cardiac life support. In Singapore, the IBLS/CPR courses are taught as part of the Basic Cardiac Life Support (BCLS) course, which is based on the Singapore Resuscitation Guidelines ${ }^{(10)}$ and uses a combination of didactic lectures, video screenings and manikin demonstrations.

We found that most of the recent studies that examined adult knowledge, attitudes and perceptions with regard to IBLS were from countries culturally and ethnically different from

\footnotetext{
${ }^{1}$ SingHealth Polyclinics - Bukit Merah, ${ }^{2}$ Department of Neonatal and Developmental Medicine, Singapore General Hospital, ${ }^{3}$ Yong Loo Lin School of Medicine, National University of Singapore, ${ }^{4}$ SpecialKids Child Health and Development Clinic, Singapore

Correspondence: Dr Lian Wee Bin, Medical Director, SpecialKids Child Health and Development Clinic, 301 Upper Thomson Road, \#03-03/04, Thomson Plaza, Singapore 574408.wblian@specialkidsclinic.com.sg
} 
Singapore (e.g. Australia ${ }^{(11)}$ and New Zealand $\left.{ }^{(12)}\right)$. Therefore, we designed the present study to examine local parental knowledge, attitudes and perceptions of IBLS, and to compare our findings with that of previous studies. ${ }^{(11,12)}$ We anticipate our findings to provide information that could be useful to the relevant authorities that plan strategies for the early recognition of paediatric CPA, thus helping to improve the outcomes of out-of hospital paediatric CPA.

\section{METHODS}

The present study was conducted at the Neonatal Department of Singapore General Hospital (SGH), Singapore, between 1 September and 31 December 2008. Parents of infants delivered at SGH (i.e. inpatients) and those who brought their children for consultation at SGH outpatient clinics during the study period were enrolled in the present study. Care was taken to ensure that the questionnaire was not re-administered to parents who had previously been administered the questionnaire either in an inpatient or outpatient setting.

The questionnaire was developed primarily in the English language and then translated into Chinese by a native Chinesespeaking nursing staff at our department. It was translated into Malay by the first author, who had received Malay language education for 13 years in Malaysia. To cater to participants with lower education (defined as secondary school education and below) simple words were used, and the questions and answers were tailored to be short, straightforward and to the point. Pictograms were also made available for the first question in the Advance Knowledge (AK) subsection so as to reduce potential confusion among participants. The questionnaires were distributed on a daily basis in both inpatient and outpatient settings. If both parents of a child were simultaneously enrolled, they were advised to complete the questionnaire separately, constituting two participants.

The questionnaire comprised three sections. Section A focused on demographic profiling of the participant. Those who had previously attended an IBLS course were denoted as 'trained', while those who had no prior attendance were denoted as 'untrained'. Section B (knowledge section) focused on the general knowledge of participants, gauging their assessment skills (with regard to an infant's conscious state) and knowledge on seeking help for an unconscious infant. Questions in section B were subdivided into two further subsections - part 1 (Basic Knowledge [BK]) required the participation of the entire cohort, and contained questions targeting the basic understanding of critical concepts; part 2 (AK) only required responses from trained participants and targeted advanced concepts. Section C comprised questions assessing parental attitudes and perceptions regarding IBLS; this section was used to provide insight into the differences between trained and untrained participants.

The questionnaire comprised a mixture of multiple choice and true/false questions. Responses were considered invalid if the ticks fell outside the scaled boxes or more than one box was ticked. Participants who failed to indicate whether they had previously attended an IBLS course were excluded, as further comparative analysis would have been difficult with this subset of participants. One point was awarded for each correct response in section $B$. The absence of a tick was deemed to indicate ignorance, and no marks were awarded for such responses. A T-score was calculated for each section of the questionnaire. Any subsection of the questionnaire that had at least one invalid response was considered to have an invalid T-score and was therefore excluded from subsequent T-score analyses.

The knowledge section was classified into two sections for scoring - BK and AK. Total Knowledge (TK) scores (comprising the total points obtained in the BK and AK subsections) were only applied for trained participants. Pass scores for BK and TK T-scores were arbitrarily defined prior to the analysis of T-scores as T-score $\geq 7$ (above T-percentage of $75 \%$ ) and $\geq 12$ (above T-percentage of $85 \%$ ), respectively. A higher pass mark was assigned for TK T-scores, as only trained participants would have attempted the AK section.

In section $C$, the participants' responses were in the form of yes/no answers. Participants who indicated 'no' were encouraged to select the most appropriate reason from those indicated for not practising their skills.

Data was analysed using the Statistical Package for the Social Sciences for Windows version 16.0 (SPSS Inc, Chicago, IL, USA), and examined for normality of distribution. Means were compared using the Student $t$-test for normally distributed data, while medians were compared using the Mann-Whitney $U$ test and Kruskal-Wallis test for skewed data. Chi-square test was used to compare data for categorical variables. Logistic regression analysis was performed to evaluate factors (e.g. ethnicity, age, gender, education, number of children and previous BCLS attendance) that contributed to a failed T-score. A p-value of $<0.05$ was considered statistically significant.

\section{RESULTS}

A total of 400 parents participated in our survey. When the questionnaires were collected, none of the participants indicated that the questions were incomprehensible. Fig. 1 demonstrates the distribution of the study participants based on their BK T-scores. For the knowledge section, 21 participants had invalid T-scores and were excluded from further analysis. Additionally, four participants who did not indicate whether they had previously attended an IBLS course were excluded in the comparative analysis of BK and TK scores between trained and untrained participants.

The median BK T-score of the entire cohort with valid scores $(n=375)$ was 7 (range $1-9)$. Pass marks were obtained by $55.2 \%$ of the participants. Among the participants who passed, $26.1 \%$ had received previous training. The median BK T-scores were 6 (range 3-9) and 8 (range 3-9) among untrained and 


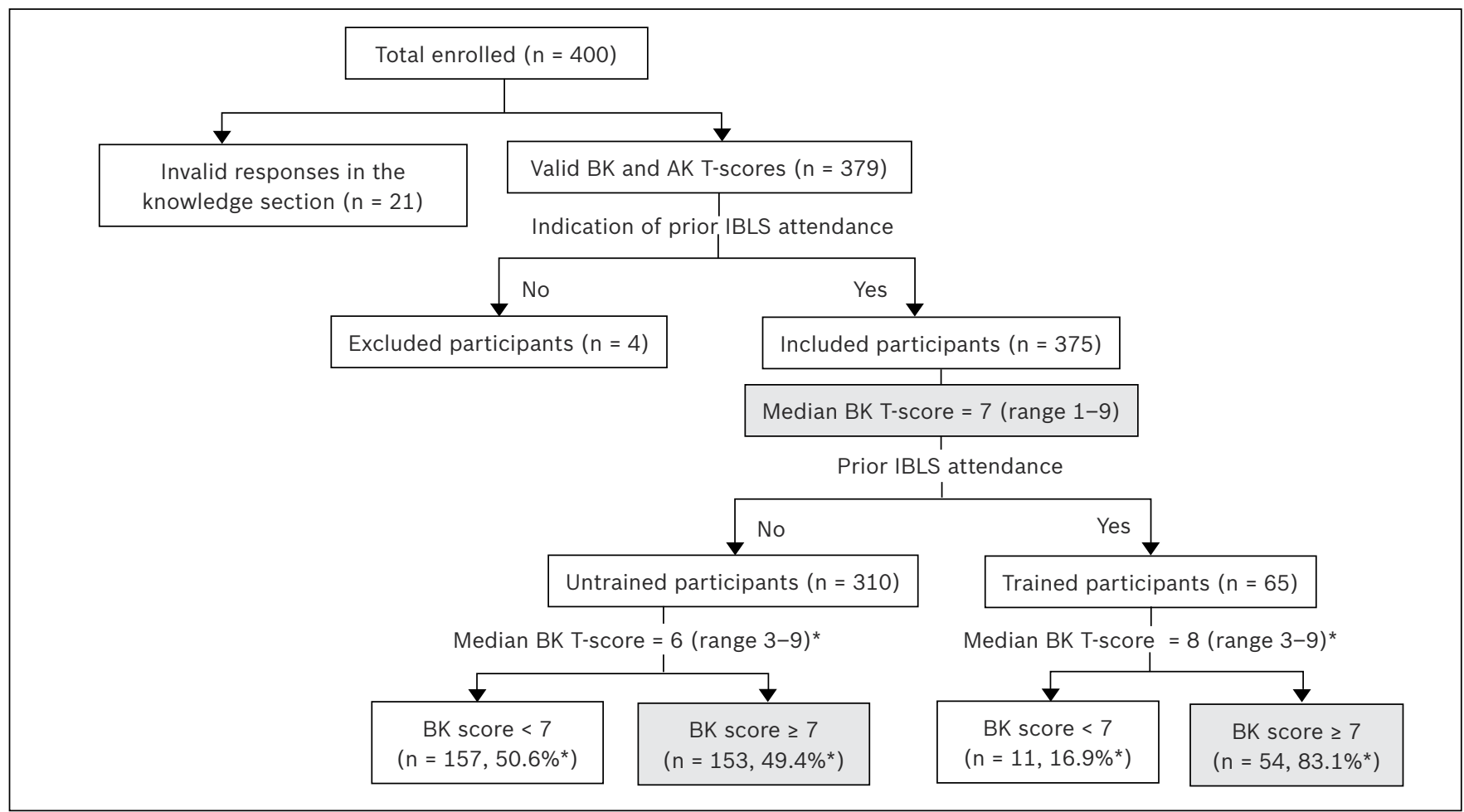

Fig. 1 Chart shows the distribution of participants based on BK T-scores. Pass mark was arbitrarily defined before analysis as BK T-score $\geq 7$ or T-percentage $>75 \%$. ${ }^{*} p<0.05$ (i.e. statistically significant). AK: Advance Knowledge; BK: Basic Knowledge; IBLS: infant basic life support

Table I. Demographic characteristics of the study cohort and the associated median Basic Knowledge (BK) T-scores and proportion of participants who obtained pass marks.

\begin{tabular}{|c|c|c|c|c|c|}
\hline \multirow[t]{2}{*}{ Characteristic } & \multirow{2}{*}{$\begin{array}{c}\text { No. of } \\
\text { participants }\end{array}$} & \multicolumn{2}{|c|}{ BK T-score } & \multicolumn{2}{|c|}{ Pass marks obtained } \\
\hline & & Median (range) & p-value & No. (\%) & p-value \\
\hline Overall & 375 & $7(1-9)$ & - & $207(55.2)$ & - \\
\hline $\begin{array}{l}\text { Ethnicity }(\mathbf{n}=\mathbf{3 7 5}) \\
\text { Chinese } \\
\text { Malay } \\
\text { Indian } \\
\text { Other }\end{array}$ & $\begin{array}{c}211 \\
106 \\
37 \\
21\end{array}$ & $\begin{array}{l}7(1-9) \\
7(1-9) \\
7(2-9) \\
7(1-8)\end{array}$ & 0.639 & $\begin{array}{r}122(57.8) \\
55(51.9) \\
19(51.4) \\
11(52.4)\end{array}$ & 0.718 \\
\hline $\begin{array}{l}\text { Age group (yrs) }(\mathbf{n}=\mathbf{3 7 4}) \\
21-25 \\
26-30 \\
31-35 \\
36-40 \\
41-60\end{array}$ & $\begin{array}{c}29 \\
112 \\
129 \\
77 \\
27\end{array}$ & $\begin{array}{l}6(3-9) \\
7(2-9) \\
7(1-9) \\
6(1-9) \\
6(2-9)\end{array}$ & $0.034^{+}$ & $\begin{array}{l}14(48.3) \\
68(60.7) \\
78(60.5) \\
33(42.9) \\
12(44.4)\end{array}$ & 0.091 \\
\hline $\begin{array}{l}\text { Gender }(\mathbf{n}=\mathbf{3 6 4}) \\
\text { Male } \\
\text { Female }\end{array}$ & $\begin{array}{l}127 \\
237\end{array}$ & $\begin{array}{l}7(1-9) \\
7(1-9)\end{array}$ & 0.757 & $\begin{array}{r}72(56.7) \\
134(56.5)\end{array}$ & 0.421 \\
\hline $\begin{array}{l}\text { Education }(\mathbf{n}=\mathbf{3 7 3}) \\
\text { Secondary or lower } \\
\text { Tertiary or higher }\end{array}$ & $\begin{array}{c}91 \\
282\end{array}$ & $\begin{array}{l}6(1-9) \\
7(1-9)\end{array}$ & $<0.001^{\dagger}$ & $\begin{array}{r}38(41.8) \\
168(59.6)\end{array}$ & $0.012^{+}$ \\
\hline $\begin{array}{l}\text { No. of children }(n=375) \\
1 \\
2 \\
3 \\
4 \\
>4\end{array}$ & $\begin{array}{c}185 \\
110 \\
55 \\
13 \\
12\end{array}$ & $\begin{array}{l}7(1-9) \\
7(1-9) \\
7(1-9) \\
6(3-8) \\
7(3-8)\end{array}$ & 0.945 & $\begin{array}{r}104(56.2) \\
57(51.8) \\
32(58.2) \\
5(38.5) \\
9(75.0)\end{array}$ & 0.385 \\
\hline
\end{tabular}

Participants for whom data was not known were excluded; only those with valid responses and valid T-scores were included for analysis. ${ }^{\dagger} \mathrm{p}<0.05$ (i.e. statistically significant).

trained participants, respectively $(\mathrm{p}<0.001)$. The proportion of participants who had a BK T-score $\geq 7$ was significantly higher among the trained participants.

Table I shows the demographic characteristics of our study cohort, and the median BK T-scores and proportion of participants who obtained pass marks with respect to these characteristics. Median BK T-scores were significantly higher among participants in the age groups 26-30 years and 31-35 years when compared to other age groups. Nearly $75.6 \%$ of the participants had received a tertiary education or higher. 
Table II. Median Basic Knowledge (BK) T-scores and proportion of participants who obtained pass marks among untrained participants.

\begin{tabular}{|c|c|c|c|c|c|}
\hline \multirow[t]{2}{*}{ Characteristic } & \multirow{2}{*}{$\begin{array}{c}\text { No. of } \\
\text { participants }\end{array}$} & \multicolumn{2}{|c|}{ BK T-score } & \multicolumn{2}{|c|}{ Pass marks obtained* } \\
\hline & & Median (range) & p-value & No. (\%) & p-value \\
\hline Untrained participants & 310 & $6(3-9)$ & - & $153(49.4)$ & - \\
\hline $\begin{array}{l}\text { Ethnicity }(\mathbf{n}=\mathbf{3 1 0}) \\
\text { Chinese } \\
\text { Malay } \\
\text { Indian } \\
\text { Other }\end{array}$ & $\begin{array}{l}178 \\
85 \\
30 \\
17\end{array}$ & $\begin{array}{l}7(1-9) \\
6(1-8) \\
6(2-8) \\
6(1-8)\end{array}$ & 0.218 & $\begin{array}{r}95(53.4) \\
37(43.5) \\
13(43.3) \\
8(47.1)\end{array}$ & 0.428 \\
\hline $\begin{array}{l}\text { Age group (yrs) }(\mathbf{n}=\mathbf{3 1 0}) \\
21-25 \\
26-30 \\
31-35 \\
36-40 \\
41-60\end{array}$ & $\begin{array}{c}24 \\
87 \\
105 \\
70 \\
24\end{array}$ & $\begin{array}{l}6(3-8) \\
7(2-9) \\
7(1-9) \\
6(1-9) \\
6(2-9)\end{array}$ & 0.134 & $\begin{array}{r}9(37.5) \\
45(51.7) \\
59(56.2) \\
29(41.4) \\
11(45.8)\end{array}$ & 0.250 \\
\hline $\begin{array}{l}\text { Gender }(\mathbf{n}=\mathbf{3 0 2}) \\
\text { Male } \\
\text { Female }\end{array}$ & $\begin{array}{l}107 \\
195\end{array}$ & $\begin{array}{l}7(1-9) \\
6(1-9)\end{array}$ & 0.475 & $\begin{array}{l}57(53.3) \\
91(46.7)\end{array}$ & 0.412 \\
\hline $\begin{array}{l}\text { Education }(\mathbf{n}=\mathbf{3 0 8}) \\
\text { Secondary or lower } \\
\text { Tertiary or higher }\end{array}$ & $\begin{array}{c}80 \\
228\end{array}$ & $\begin{array}{l}6(1-9) \\
7(1-9)\end{array}$ & $0.001^{+}$ & $\begin{array}{r}30(37.5) \\
122(53.5)\end{array}$ & $0.048^{+}$ \\
\hline $\begin{array}{l}\text { No. of children }(\mathbf{n}=\mathbf{3 1 0}) \\
1 \\
2 \\
3 \\
4 \\
>4\end{array}$ & $\begin{array}{c}149 \\
92 \\
47 \\
11 \\
11\end{array}$ & $\begin{array}{l}6(1-9) \\
6(1-9) \\
7(1-9) \\
6(3-8) \\
7(3-8)\end{array}$ & 0.731 & $\begin{array}{r}72(48.3) \\
42(45.7) \\
27(57.4) \\
4(36.4) \\
8(72.7)\end{array}$ & 0.293 \\
\hline
\end{tabular}

Participants for whom data was not known were excluded; only those with valid responses and valid T-scores were included for analysis. ${ }^{\star}$ Pass mark was arbitrarily defined before analysis as BK T-score $\geq 7$ or T-percentage $>75 \% .{ }^{\dagger} p<0.05$ (i.e. statistically significant).

The median BK T-score and proportion of participants who obtained pass marks were consistently and significantly higher among participants with higher educational qualifications.

The median BK T-score and proportion of participants who obtained pass marks among untrained participants are shown in Table II. A significant difference was detected only with respect to levels of education; participants who had a tertiary education or higher achieved a higher median BK T-score, and a larger proportion of them obtained pass marks. The median BK T-scores and percentage of participants who obtained pass marks among trained participants are shown in Table III. A majority ( $n=54,83.1 \%$ ) of the trained participants obtained pass marks with a median BK T-score of 8 (range 3-9). There was no statistically significant difference in the scores of trained participants with respect to the various demographic characteristics.

Among the trained participants, the median TK T-score was 11 (range 3-14) and pass marks were obtained by only $35.4 \%$ of them (Table IV). The median TK T-scores and proportion of participants who obtained pass marks were significantly higher among participants with higher educational qualifications. There was no statistically significant difference in the scores of these participants with respect to other demographic characteristics such as ethnicity, age group, gender and number of children.

When the median BK T-scores of trained and untrained participants (Table V) were compared, significant differences were observed in the two groups with respect to ethnicity, younger age, gender, education and parents with one or two children. Logistic regression analysis for the various factors (such as non-Chinese ethnicity, male gender, secondary education or lower, having two or more children and lack of previous training) revealed that for BK T-scores of the entire cohort, age of participants $\geq 35$ years (odds ratio [OR] 1.825, 95\% confidence interval $[\mathrm{Cl}] 1.109-3.005 ; \mathrm{p}=0.018)$, secondary education or lower (OR 2.207, 95\% Cl 1.291-3.773; $\mathrm{p}=0.004$ ) and absence of previous BCLS training (OR 4.381, 95\% Cl 2.167-8.859; $\mathrm{p}<0.001)$ were significant factors that contributed toward failure in achieving BK T-score pass marks (data not shown). For TK T-scores among trained participants, none of these factors were found to be significant (data not shown).

When individual questions were considered, trained participants were found to be able to answer most BK questions correctly except for question $4 \mathrm{a}$, for which only $38.0 \%$ responded correctly (Table VI). Notably, the number of participants who responded correctly to this question among trained participants was significantly higher than that among untrained participants (21.0\%). All questions, except for questions 1 and $5 \mathrm{a}$, showed significantly higher rates of correct responses among trained participants. About $13.6 \%$ of participants did not know the telephone number to call in an emergency.

For AK questions, trained participants obtained pass marks in four out of five questions, but for two of these questions, the proportion of participants who responded correctly was only slightly over $50 \%$ (Table VII). The proportion of participants 
Table III. Median Basic Knowledge (BK) T-scores and proportion of participants who obtained pass marks among trained participants.

\begin{tabular}{|c|c|c|c|c|c|}
\hline \multirow[t]{2}{*}{ Characteristic } & \multirow{2}{*}{$\begin{array}{c}\text { No. of } \\
\text { participants }\end{array}$} & \multicolumn{2}{|c|}{ BK T-score } & \multicolumn{2}{|c|}{ Pass marks obtained* } \\
\hline & & Median (range) & p-value & No. (\%) & p-value \\
\hline Trained participants & 65 & $8(3-9)$ & - & $54(83.1)$ & - \\
\hline $\begin{array}{l}\text { Ethnicity }(\mathbf{n}=\mathbf{6 5}) \\
\text { Chinese } \\
\text { Malay } \\
\text { Indian } \\
\text { Other }\end{array}$ & $\begin{array}{l}33 \\
21 \\
7 \\
4\end{array}$ & $\begin{array}{l}7(5-9) \\
8(6-9) \\
8(6-9) \\
8(3-8)\end{array}$ & 0.593 & $\begin{array}{r}27(81.8) \\
18(85.7) \\
6(85.7) \\
3(75.0)\end{array}$ & 0.948 \\
\hline $\begin{array}{l}\text { Age group }(\mathbf{y r s})(\mathbf{n}=\mathbf{6 4}) \\
21-25 \\
26-30 \\
31-35 \\
36-40 \\
41-60\end{array}$ & $\begin{array}{c}5 \\
25 \\
24 \\
7 \\
3\end{array}$ & $\begin{array}{l}9(7-9) \\
8(5-9) \\
7(3-9) \\
7(6-9) \\
7(6-8)\end{array}$ & 0.064 & $\begin{aligned} 5 & (100.0) \\
23 & (92.0) \\
19 & (79.2) \\
4 & (57.1) \\
2 & (66.7)\end{aligned}$ & 0.234 \\
\hline $\begin{array}{l}\text { Gender }(\mathbf{n}=\mathbf{6 2}) \\
\text { Male } \\
\text { Female }\end{array}$ & $\begin{array}{l}20 \\
42\end{array}$ & $\begin{array}{l}8(5-9) \\
8(3-9)\end{array}$ & 0.607 & $\begin{array}{l}15(75.0) \\
36(85.7)\end{array}$ & 0.418 \\
\hline $\begin{array}{l}\text { Education }(\mathbf{n}=\mathbf{6 5}) \\
\text { Secondary or lower } \\
\text { Tertiary or higher }\end{array}$ & $\begin{array}{l}11 \\
54\end{array}$ & $\begin{array}{l}7(6-9) \\
8(3-9)\end{array}$ & 0.204 & $\begin{array}{r}8(72.7) \\
46(85.2)\end{array}$ & 0.315 \\
\hline $\begin{array}{l}\text { No. of children }(n=65) \\
1 \\
2 \\
3 \\
4 \\
>4\end{array}$ & $\begin{array}{c}36 \\
18 \\
8 \\
2 \\
1\end{array}$ & $\begin{array}{l}8(5-9) \\
8(5-9) \\
7(3-8) \\
7(6-7) \\
-\end{array}$ & 0.225 & $\begin{aligned} 36 & (100.0) \\
15 & (83.3) \\
5 & (62.5) \\
1 & (50.0) \\
1 & (100.0)\end{aligned}$ & 0.284 \\
\hline
\end{tabular}

Participants for whom data was not known were excluded; only those with valid responses and valid T-scores were included for analysis. ${ }^{*}$ Pass mark was arbitrarily defined before analysis as BK T-score $\geq 7$ or T-percentage $>75 \%$.

Table IV. Total Knowledge (TK) T-scores and proportion of participants who obtained pass marks among trained participants.

\begin{tabular}{|c|c|c|c|c|c|}
\hline \multirow[t]{2}{*}{ Characteristic } & \multirow{2}{*}{$\begin{array}{c}\text { No. of } \\
\text { participants }\end{array}$} & \multicolumn{2}{|c|}{ TK T-score } & \multicolumn{2}{|c|}{ Pass marks obtained* } \\
\hline & & Median (range) & p-value & No. (\%) & p-value \\
\hline Trained participants & 65 & $11(3-14)$ & - & $23(35.4)$ & - \\
\hline $\begin{array}{l}\text { Ethnicity }(\mathbf{n}=\mathbf{6 5}) \\
\text { Chinese } \\
\text { Malay } \\
\text { Indian } \\
\text { Other }\end{array}$ & $\begin{array}{c}33 \\
21 \\
7 \\
4\end{array}$ & $\begin{array}{l}10(8-14) \\
11(6-14) \\
11(8-14) \\
11(3-12)\end{array}$ & 0.760 & $\begin{aligned} 10 & (30.3) \\
10 & (47.6) \\
2 & (28.6) \\
1 & (25.0)\end{aligned}$ & 0.556 \\
\hline $\begin{array}{l}\text { Age group (yrs) }(\mathbf{n}=\mathbf{6 4}) \\
21-25 \\
26-30 \\
31-35 \\
36-40 \\
41-60\end{array}$ & $\begin{array}{c}5 \\
25 \\
24 \\
7 \\
3\end{array}$ & $\begin{aligned} 11 & (10-14) \\
11 & (8-14) \\
10 & (3-13) \\
9 & (8-14) \\
1 & (10-12)\end{aligned}$ & 0.074 & $\begin{aligned} 2 & (40.0) \\
12 & (48.0) \\
7 & (29.2) \\
1 & (14.3) \\
1 & (33.3)\end{aligned}$ & 0.534 \\
\hline $\begin{array}{l}\text { Gender }(\mathbf{n}=\mathbf{6 2}) \\
\text { Male } \\
\text { Female }\end{array}$ & $\begin{array}{l}20 \\
42\end{array}$ & $\begin{array}{l}10(6-14) \\
11(3-14)\end{array}$ & 0.239 & $\begin{array}{r}6(30.0) \\
17(40.5)\end{array}$ & 0.305 \\
\hline $\begin{array}{c}\text { Education }(\mathbf{n}=\mathbf{6 5}) \\
\text { Secondary or lower } \\
\text { Tertiary or higher }\end{array}$ & $\begin{array}{l}11 \\
54\end{array}$ & $\begin{array}{l}10(8-14) \\
11(3-14)\end{array}$ & $0.015^{+}$ & $\begin{array}{cl}1 & (9.1) \\
22 & (40.7)\end{array}$ & $0.045^{+}$ \\
\hline $\begin{array}{l}\text { No. of children }(n=65) \\
1 \\
2 \\
3 \\
4 \\
>4\end{array}$ & $\begin{array}{c}36 \\
18 \\
8 \\
2 \\
1\end{array}$ & $\begin{array}{l}11(8-14) \\
11(8-14) \\
10(3-11) \\
10(9-10) \\
\quad-\end{array}$ & 0.058 & $\begin{aligned} & 16(44.4) \\
& 7(38.9) \\
& 0(0) \\
& 0(0) \\
& 0(0)\end{aligned}$ & 0.116 \\
\hline
\end{tabular}

Participants for whom data was not known were excluded; only those with valid responses and valid T-scores were included for analysis. ${ }^{*}$ Pass mark was arbitrarily defined before analysis as TK T-score $\geq 12$ or T-percentage $>85 \%$. ${ }^{\dagger} p<0.05$ (i.e. statistically significant). 
Table V. Comparison of median Basic Knowledge (BT) T-scores and proportion of participants who obtained pass marks between untrained and trained participants.

\begin{tabular}{|c|c|c|c|c|c|c|c|c|c|}
\hline \multirow[t]{2}{*}{ Characteristic } & \multicolumn{3}{|c|}{ No. of participants } & \multicolumn{3}{|c|}{ Median BK T-score (range) } & \multicolumn{3}{|c|}{ No. participants with pass marks } \\
\hline & Total & Untrained & Trained & Untrained & Trained & p-value & Untrained & Trained & p-value \\
\hline Trained participants & 375 & 310 & 65 & $6(3-9)$ & $8(3-9)$ & $<0.001^{*}$ & & & \\
\hline \multicolumn{10}{|l|}{ Ethnicity (n = 375) } \\
\hline Chinese & 211 & 178 & 33 & $7(1-9)$ & $7(5-9)$ & $<0.001^{*}$ & 95 & 27 & $0.002^{*}$ \\
\hline Malay & 106 & 85 & 21 & $6(1-8)$ & $8(6-9)$ & $<0.001^{*}$ & 37 & 18 & $0.001^{*}$ \\
\hline Indian & 37 & 30 & 7 & $6(2-8)$ & $8(6-9)$ & $0.010^{*}$ & 13 & 6 & $0.043^{*}$ \\
\hline \multicolumn{10}{|c|}{ Age group (yrs) $(n=374)$} \\
\hline $21-25$ & 29 & 24 & 5 & $6(3-8)$ & $9(7-9)$ & $0.002^{*}$ & 9 & 5 & $0.011^{*}$ \\
\hline $26-30$ & 112 & 87 & 25 & $7(2-9)$ & $8(5-9)$ & $<0.001^{*}$ & 45 & 23 & $<0.001^{*}$ \\
\hline $31-35$ & 129 & 105 & 24 & $7(1-9)$ & $7(3-9)$ & $0.019 *$ & 59 & 19 & 0.038 \\
\hline $36-40$ & 77 & 70 & 7 & $6(1-9)$ & $7(6-9)$ & 0.066 & 29 & 4 & 0.423 \\
\hline $41-60$ & 27 & 24 & 3 & $6(2-9)$ & $7(6-8)$ & 0.437 & 11 & 2 & 0.496 \\
\hline \multicolumn{10}{|l|}{ Gender $(n=364)$} \\
\hline Female & 237 & 195 & 42 & $6(1-9)$ & $8(3-9)$ & $<0.001^{*}$ & 91 & 36 & $<0.001^{*}$ \\
\hline \multicolumn{10}{|l|}{ Education ( $n=373$ ) } \\
\hline Secondary or lower & 91 & 80 & 11 & $6(1-9)$ & $7(6-9)$ & $0.004^{*}$ & 30 & 8 & $0.026^{*}$ \\
\hline Tertiary or higher & 282 & 228 & 54 & $7(1-9)$ & $8(3-9)$ & $<0.001^{*}$ & 122 & 46 & $<0.001^{*}$ \\
\hline \multicolumn{10}{|c|}{ No. of children $(n=375)$} \\
\hline 1 & 185 & 149 & 36 & $6(1-9)$ & $8(5-9)$ & $<0.001^{*}$ & 72 & 36 & $<0.001^{*}$ \\
\hline 2 & 110 & 92 & 18 & $6(1-9)$ & $8(5-9)$ & $<0.001^{*}$ & 42 & 15 & $0.003^{*}$ \\
\hline 3 & 55 & 47 & 8 & $7(1-9)$ & $7(3-8)$ & 0.519 & 27 & 5 & 0.789 \\
\hline 4 & 13 & 11 & 2 & $6(3-8)$ & $7(6-7)$ & 0.769 & 4 & 1 & 0.715 \\
\hline$>4$ & 12 & 11 & 1 & $7(3-8)$ & - & - & 8 & 1 & 0.546 \\
\hline
\end{tabular}

Participants for whom data was not known were excluded; only those with valid responses and valid T-scores were included for analysis. ${ }^{*} \mathrm{p}<0.05$ (i.e. statistically significant).

Table VI. Comparison of median Basic Knowledge (BT) T-scores and proportion of participants who obtained pass marks between untrained and trained participants.

\begin{tabular}{|c|c|c|c|c|c|}
\hline \multirow[t]{2}{*}{ Question } & \multirow[t]{2}{*}{ Correct response } & \multicolumn{3}{|c|}{$\%$ of participants with correct responses } & \multirow[t]{2}{*}{ p-value } \\
\hline & & Total & Untrained & Trained & \\
\hline $\begin{array}{l}\text { 1. What is the Singapore } \\
\text { ambulance access number? }\end{array}$ & 995 & 86.4 & 85.0 & 92.0 & 0.163 \\
\hline $\begin{array}{l}\text { 2. How long can a child's brain live } \\
\text { without oxygen (breathing)? }\end{array}$ & $4-6$ mins & 68.8 & 66.0 & 81.0 & $0.018^{*}$ \\
\hline $\begin{array}{l}\text { 3. What is the first thing that you } \\
\text { will do when you find your child } \\
\text { unconscious or unresponsive? }\end{array}$ & $\begin{array}{l}\text { Try to wake the child up, and } \\
\text { if he/she does not show any } \\
\text { response immediately, or within } \\
30 \text { s, start CPR for } 2 \text { mins, and } \\
\text { then check airway, breathing } \\
\text { and circulation }\end{array}$ & 67.7 & 63.0 & 89.0 & $<0.001^{*}$ \\
\hline \multicolumn{6}{|l|}{$\begin{array}{l}\text { 4. I can try to wake an unconscious } \\
\text { infant by: }\end{array}$} \\
\hline a. Calling the infant repeatedly. & False & 24.3 & 21.0 & 38.0 & $0.006^{*}$ \\
\hline $\begin{array}{l}\text { b. Tapping or gently shaking the } \\
\text { infant's shoulder. }\end{array}$ & True & 85.9 & 84.0 & 94.0 & $0.049^{*}$ \\
\hline $\begin{array}{l}\text { c. Putting a very cold cloth on the } \\
\text { infant's body. }\end{array}$ & False & 59.5 & 56.0 & 74.0 & $0.012^{*}$ \\
\hline \multicolumn{6}{|l|}{$\begin{array}{l}\text { 5. How do you to confirm that a child } \\
\text { is not breathing when he/she is } \\
\text { unconscious? }\end{array}$} \\
\hline $\begin{array}{l}\text { a. By looking for rise and fall of } \\
\text { the chest. }\end{array}$ & True & 88.5 & 87.0 & 94.0 & 0.197 \\
\hline $\begin{array}{l}\text { b. By listening for air escaping } \\
\text { during exhalation. }\end{array}$ & True & 78.9 & 76.0 & 94.0 & $0.001^{*}$ \\
\hline $\begin{array}{l}\text { c. By feeling for flow of air from } \\
\text { the infant's mouth and nose } \\
\text { using my cheek. }\end{array}$ & True & 83.5 & 82.0 & 92.0 & $0.042^{*}$ \\
\hline
\end{tabular}


Table VII. Responses to the Advance Knowledge section ( $\mathbf{n}=65$ ).

\begin{tabular}{|c|c|c|}
\hline Question & $\begin{array}{l}\text { Correct } \\
\text { response }\end{array}$ & $\begin{array}{l}\% \text { of participants } \\
\text { with correct } \\
\text { responses }\end{array}$ \\
\hline \multicolumn{3}{|l|}{$\begin{array}{l}\text { 1. If you witness a conscious child } \\
\text { unable to breathe and choking } \\
\text { from a piece of toy he/she has } \\
\text { put into his/her mouth, what } \\
\text { should you do? }\end{array}$} \\
\hline $\begin{array}{l}\text { a. Use your finger to sweep } \\
\text { the mouth despite being } \\
\text { unable to see the toy. }\end{array}$ & False & 53.6 \\
\hline $\begin{array}{l}\text { b. Lie the child immediately } \\
\text { on my thigh face down, } \\
\text { deliver } 5 \text { back blows, then } \\
\text { turn him/her over onto his/ } \\
\text { her back resting on my thigh } \\
\text { and deliver } 5 \text { chest thrust. }\end{array}$ & True & 91.3 \\
\hline $\begin{array}{l}\text { c. Turn the child upside down } \\
\text { and shake him/her in an } \\
\text { up- and downward action } \\
\text { repeatedly. }\end{array}$ & False & 91.3 \\
\hline $\begin{array}{l}\text { 2. What is the ratio of chest } \\
\text { compression to effective } \\
\text { breaths when performing } \\
\text { CPR on a child? }\end{array}$ & $30: 2$ & 29.0 \\
\hline $\begin{array}{l}\text { 3. The location to check for a } \\
\text { pulse in a child aged } \\
<1 \text { year old is the neck }\end{array}$ & False & 52.2 \\
\hline
\end{tabular}

CPR: cardiopulmonary resuscitation

Table VIII. Attitudes and perceptions among untrained participants.

\begin{tabular}{ll}
\hline Question and answer & No. (\%)* \\
\hline $\begin{array}{l}\text { 1. Will you attend the IBLS course if it is } \\
\text { offered to you? }\end{array}$ & $267(84.8)$ \\
Yes & $48^{+}(15.2)$ \\
No & \\
Reasons for refusal & $10^{+}$ \\
i. I know what to do in an & $20^{+}$ \\
$\quad$ emergency situation & $8^{+}$ \\
ii. No time & $2^{+}$ \\
iii. Financial difficulty & \\
iv. Other reasons & \\
2. Should this course be offered to all & $296(94.0)$ \\
parents? & $19(6.0)$ \\
Yes &
\end{tabular}

${ }^{*}$ Participants who did not state whether they attended any infant basic life support (IBLS) courses were excluded from the analysis. ${ }^{\dagger} T h e$ entries do not add up as some participants did not respond to the questions. For those who did, not all gave their reason.

who responded correctly was the lowest for question 2, which pertained to the ratio of chest compressions to effective breaths when performing CPR on a child. Changes were instituted for the ratio in Singapore in 2006 as part of the National Resuscitation Guidelines. ${ }^{(13)}$

Among the 51 participants who indicated the year they attended the IBLS course, $70.6 \%$ had been trained after 2005 (median year of training was 2007). There is a positive trend among trained participants who attempted question 2. More participants who took the IBLS course after 2005 achieved pass marks $(11 / 36,30.6 \%)$ for this question than those who took the IBLS course prior to 2006 (2/15, 13.3\%). With regard to what they would do in an emergency, $20.1 \%$ of participants
Table IX. Attitudes and perceptions among trained participants.

\begin{tabular}{|c|c|}
\hline Question and answer & No. $(\%)^{*}$ \\
\hline \multicolumn{2}{|l|}{$\begin{array}{l}\text { 1. Will you be interested to attend a } \\
\text { refresher IBLS course every } 4-5 \text { years? }\end{array}$} \\
\hline Yes & $50(87.7)$ \\
\hline No & $7^{+}(12.3)$ \\
\hline \multicolumn{2}{|l|}{ Reasons for refusal } \\
\hline $\begin{array}{l}\text { i. I know what to do in an } \\
\text { emergency situation }\end{array}$ & $1^{+}$ \\
\hline ii. No time & $1^{+}$ \\
\hline iii. Financial difficulty & $0^{+}$ \\
\hline iv. Other reasons & $1^{+}$ \\
\hline \multicolumn{2}{|l|}{$\begin{array}{l}\text { 2. Will you use your IBLS skills if your child } \\
\text { suddenly goes into cardiopulmonary arrest } \\
\text { or is choking from a foreign object? }\end{array}$} \\
\hline Yes & $54(93.1)$ \\
\hline No & $4^{+}(6.0)$ \\
\hline \multicolumn{2}{|l|}{ Reasons for refusal } \\
\hline $\begin{array}{l}\text { i. Lack of confidence and afraid } \\
\text { to cause more harm }\end{array}$ & $1^{+}$ \\
\hline $\begin{array}{l}\text { ii. I can reach the hospital fast enough } \\
\text { where expert help is better }\end{array}$ & $1^{+}$ \\
\hline iii. Other reasons & $0^{+}$ \\
\hline \multicolumn{2}{|l|}{$\begin{array}{l}\text { 2. If you see another child in } \\
\text { cardiopulmonary arrest, will you use } \\
\text { your IBLS skills to help him/her? }\end{array}$} \\
\hline Yes & $50(86.2)$ \\
\hline No & $8^{+}(13.8)$ \\
\hline \multicolumn{2}{|l|}{ Reasons for refusal } \\
\hline $\begin{array}{l}\text { i. Lack of confidence and afraid to } \\
\text { cause more harm }\end{array}$ & $3^{+}$ \\
\hline $\begin{array}{l}\text { ii. Afraid of adverse legal actions from } \\
\text { the child's parents if the child dies/ } \\
\text { develops hypoxic brain injury }\end{array}$ & $4^{+}$ \\
\hline iii. Not my concern & $0^{+}$ \\
\hline iv. Other reasons & $0^{+}$ \\
\hline
\end{tabular}

*Participants who did not state whether they attended any infant basic life support (IBLS) courses were excluded from the analysis. ${ }^{\dagger}$ The entries do not add up as some participants did not respond to the questions. For those who did, not all gave their reason

indicated that they would use their own/public transport to travel to the nearest hospital, while $3.7 \%$ would call someone for help and $8.5 \%$ would call for an ambulance and wait for assistance (data not shown). Comparing the TK scores of trained participants who took the IBLS course prior to, during and following 2007, there was a positive trend for a higher pass rate for TK scores among the participants who took the IBLS course in later years $(42.9 \%$ in $2008 ; 29.4 \%$ in $2007 ; 25.0 \%$ before 2007).

Tables VIII and IX show the attitudes and perceptions of the untrained and trained participants, respectively. Among the untrained participants, nearly $85 \%$ of the cohort indicated an interest in attending IBLS courses (Table VIII). Among the uninterested participants ( $n=48), 20(41.7 \%)$ indicated the reason for refusal as having 'no time', while 10 (20.8\%) indicated that they knew what to do in an emergency and 8 $(16.7 \%)$ indicated financial difficulty.

Among the trained participants, nearly $88 \%$ indicated interest in attending refresher IBLS courses every 4-5 years (Table IX). Only seven participants indicated a lack of such interest, citing reasons such as knowing what to do in an 
emergency $(n=1)$ and lack of time $(n=1)$. Among the four participants who indicated that they would not use their IBLS skills on their own offspring, only two participants indicated a reason, which included a lack of confidence $(n=1)$ and the ability to reach expert help at the hospital quickly $(n=1)$. Eight participants indicated that they would not use their IBLS skills on other children - four participants were afraid of adverse legal actions should the victim die or develop hypoxic brain injury, and three participants indicated a lack of confidence.

\section{DISCUSSION}

The understanding of sudden CPA as a public health problem has paralleled the development of the modern emergency medical services (EMS) system. The critical links in the paediatric chain of survival include recognition and early EMS activation, early CPR with emphasis on chest compressions, defibrillation, early advanced life support and integrated postcardiac arrest care. The chance of survival in children with sudden CPA when CPR and defibrillation are applied within four minutes is reportedly $43 \% .{ }^{(14)}$ This is reduced by $7 \%-10 \%$ with every passing minute. Despite this important chain of survival, a local study has found that EMS was activated for only $63.7 \%$ of patients who presented with out-of-hospital CPA. ${ }^{(7)}$

Several studies have been conducted to identify the factors responsible for the low survival rates associated with outof-hospital paediatric CPA. ${ }^{(7,9)}$ Tham and Chan reported that witnessed CPA was a key independent predictor of return of spontaneous circulation (OR 3.049, 95\% Cl 1.101-8.444). ${ }^{(7)}$ Furthermore, according to Swor et al, witnessed CPA was a predictor of CPR performance (OR 2.3, 95\% Cl 1.4-3.8). ${ }^{(9)}$ Although CPA is unpredictable and a witness cannot always be guaranteed, what would make a difference for the affected person would be the activation of EMS and initiation of CPR for all events that happen to be witnessed. However, bystander CPR rates, which have been shown to more than double patient survival rates, are often low, even when CPA is witnessed. ${ }^{(15)}$ Given that Swor et al have reported that CPR-trained bystanders make a significant difference to CPR performance (OR 6.6, 95\% Cl 3.5-12.5), ${ }^{(9)}$ it is evident that the understanding and experience of bystanders who may potentially witness a CPA event is crucial.

The present study aimed to assess the knowledge, attitudes and perceptions of a select group of potential bystanders in Singapore. Parents of newborn infants or children were selected as the target population, as they were most likely to be witnesses in paediatric CPA events. Our findings draw attention to several factors that are likely to have a bearing on CPR performance in paediatric CPA.

Firstly, general knowledge regarding IBLS was poor in our local cohort. However, it was found that knowledge of IBLS was significantly better among those who had previously attended IBLS training, as indicated by the higher proportion of participants who obtained pass marks and higher BK T-scores among trained participants. Our findings indicated successful retention of knowledge among trainees to a certain degree.

In our study, while expected associations were found between knowledge and training across factors such as ethnicity, gender and education, we found that prior training makes a significant difference to knowledge among participants belonging to younger age groups. In our cohort, higher scores were achieved among participants aged 26-35 years. We also found that participants over 35 years had a 2.2-fold greater risk of failing when compared to younger participants. Healthcare providers should therefore focus on this target population and promote relevant training among the younger public.

Furthermore, training appeared to be a significant factor among participants with fewer children (either one or two children). However, logistic regression analysis did not reveal any significance for those with fewer than four, three or two children. Given these findings, more widespread and targeted attempts to raise awareness, particularly among parents with fewer children (and possibly new parents), who might perhaps have more time to attend training when compared to those with more offspring, should be explored.

Logistic regression analysis revealed that training was the most important factor associated with knowledge of IBLS in our cohort, as the increased risk of failing to obtain pass marks for BK T-scores was greater than fourfold for participants without prior training. Among the trained participants, none of the other demographic factors had any significant impact on the ability to achieve pass marks for TK T-scores. Our findings imply that training is a key factor that could benefit a majority of the general population and should therefore be made easily available.

Although a majority of trained participants obtained pass marks for BK T-scores, our survey highlighted deficits in more advanced knowledge - the pass rate for TK T-scores was low at $35.4 \%$ - especially with regard to knowledge pertaining to recent updates in guidelines, such as the chest compression-tobreath ratio when performing CPR on a child. The higher scores and pass rates among participants with higher educational qualifications in our study were consistent with the findings of Swor et al, who reported that CPR performance was better among participants with high school education and higher (OR 2.0, 95\% Cl 1.2-3.1). ${ }^{(9)}$ Short refresher IBLS courses that allow continual upgrading of knowledge for participants through either practical workshops or online sessions (the latter option may be particularly useful for those who are more internetsavvy or have higher educational qualifications) should be considered. We found that all trained participants had attended their last BCLS course within the preceding ten years. This finding was in agreement with that of Swor et al, who reported that CPR training within five years was a significant predictor of CPR performance (OR 4.5, 95\% Cl 2.8-7.3). ${ }^{(9)}$

A weakness in the chain of survival following paediatric CPA that became evident in our study was a delay in EMS activation. About $14 \%$ of our participants were unaware of 
the local ambulance access number to call in the event of an emergency. Additionally, 19\% of participants indicated that they would use their own/public transport to get to the nearest hospital. We also found that knowledge that a child's brain would only permit a downtime of 4-6 minutes before the onset of hypoxic damage was significantly different between the trained and untrained participants in our study. According to a 2003 cardiac arrest and resuscitation epidemiology (CARE) study in Singapore, ${ }^{\left({ }^{8}\right)}$ the mean ambulance response time was $10.2 \pm 4.3$ mins. Such response times would allow and necessitate the implementation of CPR while witnesses wait for help to arrive. It is also noteworthy that it is likely harder for bystanders to apply CPR while travelling by own/public transport.

In our survey, a majority of the participants had not attended any IBLS or CPR courses. However, it was heartening that, among the untrained participants, a majority expressed interest in attending training sessions. A majority of the trained participants also wished to attend refresher courses every 4-5 years. On the other hand, several participants indicated that they would not use their IBLS skills on either their own or others' offspring. Some of the reasons cited for such refusal included a lack of confidence and fear of litigation. Healthcare providers should take note of and address such reservations when designing training sessions, as regular refresher courses may help participants gain more confidence and thus be more proactive as bystanders in the event of paediatric CPA.

In conclusion, our findings draw attention to the knowledge, attitudes and perceptions of parents in Singapore toward IBLS. Our results will help healthcare providers to design more effective future programmes, and improve early activation of EMS and early CPR performance by bystanders. This will in turn improve the survival rate of out-of-hospital paediatric CPA. Out-of-hospital paediatric CPA, although very rare, has a poor prognosis. Early CPR is crucial for improving the probability of survival and reducing the morbidity associated with such events. Improved CPR performance can be ensured through the augmentation and upgrading of the knowledge and skills of the population via early BCLS training and regular refresher courses.

\section{REFERENCES}

1. Gerein RB, Osmond MH, Stiell IG, et al. What are the etiology and epidemiology of out-of-hospital pediatric cardiopulmonary arrest in Ontario, Canada? Acad Emerg Med 2006; 13:653-8.

2. Kuisma M, Suominen P, Korpela R. Paediatric out-of-hospital cardiac arrest - epidemiology and outcome. Resuscitation 1995; 30:141-50.

3. Sirbaugh PE, Pepe PE, Shook JE, et al. A prospective, population-based study of the demographics, epidemiology, management and outcome of out-of-the-hospital pediatric cardiopulmonary arrest. Ann Emerg Med 1999; 33:174-84.

4. Tress EE, Kochanek PM, Saladinao RA, Manole MD. Cardiac arrest in children. J Emerg Trauma Shock 2010; 3:267-72.

5. Atkins DL, Everson-Stewart S, Sears GK, et al. Epidemiology and outcomes from out-of-hospital cardiac arrest in children: the Resuscitation Outcomes Consortium Epistry-Cardiac Arrest. Circulation 2009; 119:1484-91.

6. Bardai A, Berdowski J, van der Werf C, et al. Incidence, causes and outcomes of out-of-hospital cardiac arrest in children. A comprehensive, prospective, population-based study in the Netherlands. J Am Coll Cardiol 2011; 57:1822-8.

7. Tham LP, Chan I. Paediatric out-of-hospital cardiac arrests: epidemiology and outcome. Singapore Med J 2005; 46:289-96.

8. Eng Hock Ong M, Chan YH, Anantharaman V, et al. Cardiac arrest and resuscitation epidemiology in Singapore (CARE I study). Prehosp Emerg Care 2003; 7:427-33.

9. Swor R, Khan I, Domeier R, et al. CPR training and CPR performance: do CPR-trained bystanders perform CPR? Acad Emerg Med 2006; 13:596-601.

10. Lim SH. Basic Cardiac Life Support: 2011 Singapore Guidelines. Singapore Med J 2011; 52:538-43.

11. Cu J, Phan P, O'Leary FM. Knowledge and attitudes towards paediatrics cardiopulmonary resuscitation among carers of patients attending the Emergency Department of the Children's Hospital at Westmead. Emerg Med Australas 2009; 21:401-6

12. Moran K, Stanley T. Toddler parents training, understanding and perceptions of CPR. Resuscitation 2011; 82:572-6.

13. Anantharaman V. National Resuscitation Council, Singapore. Advisory Statement on: Compression-only Cardio-Pulmonary Resuscitation (CPR). March 3, 2008 [online]. Available at: http://media.wix.com/ ugd/f38905_64d0cd4aa0994bcfa82e997d17bbfcbd.pdf. Accessed March 1, 2014.

14. Part 13: Pediatric Basic Life Support. 2010 American Heart Association Guidelines for Cardiopulmonary Resuscitation and Emergency Cardiovascular Care [online]. Available at: http://m.circ.ahajournals.org/ content/122/18_suppl_3/S862.full. Accessed March 1, 2014.

15. Topjian AA, Berg RA, Nadkarni VM. Pediatric cardiopulmonary resuscitation: advances in science, techniques and outcomes. Pediatrics 2008; 122:1086-98. 